ALICE/PUB 2000-11

10 May 2000

\title{
Image Compression for the Silicon Drift Detectors in the ALICE Experiment
}

\author{
G. Alberici, D. Cavagnino, P. De Remigis, G. Mazza, A. Rivetti, F. Tosello, and \\ A. Werbrouck
}

for the Alice Experiment

\begin{abstract}
The output of each Silicon Drift Detector in the Inner Tracking System, being prepared for the future ALICE Experiment on the LHC, is a type of image composed of 256 successive digitalizations from each of 256 parallel charge measuring channels. The physical quantity measured is the charge released by the passage of a highly variable number of charged particles of differing ionizations in the silicon detectors. Each accepted event contains 520 256x256 pixel images with-10 bit quantized intensity levels. Effective analysis of the physics data requires that charge deposits be accurately reconstructed both in the time direction represented by successive digitalizations in each channel, as well as in the spatial direction represented by adjacent channels. Since all the useful data, generated an average of 40 times per second, must be archived for future offline analysis, a strong compression algorithm must be applied to suppress the nonessential signals and compress the useful ones.

We describe an algorithm for the zero suppression and data compression for the Silicon Drift Detectors in the ALICE experiment, which seeks to maintain maximum precision within the limits of data transmission bandwidth, to retain two-dimensional cluster reconstructability and to statistically monitor the background. Two thresholds (high and low) are employed to facilitate understanding the charge cluster neighbourhoods. This choice also helps to suppress single high background peaks and provides a statistically cleaner sample for background monitoring. Background average and standard deviation are monitored by counting the zero signals (due to negative inputs to the ADCs) and the signals above the thresholds and then using a minimization algorithm. Background counts which overflow the small counter ranges are discarded to maintain high compression and then corrected statistically offline.
\end{abstract}


The method outlined addresses a common problem of minimum-loss data transfer common to any imaging radiation detector and, therefore, could find applications in other fields, such as medical diagnostics, in which the storage of large numbers of images is required.

First the 10-bit output of the ADCs is compressed to 8 bits using a quasi parabolic monotonic truncation which requires no conversion table, no clock cycles and in which all codes are utilised. This initial compression furnishes comparable relative errors for the different ionizations of the passing particles. The expansion to the original values for offline analysis includes a compensation for the truncated signals, which is statistically without bias.

The main algorithm is purely combinatorial and thus requires only one clock cycle to code the signals and send them on through a circular twisted barrel shifter to the output FIFO. Simulations of the circuits using FPGAs and of the compression algorithm in software are in progress. 